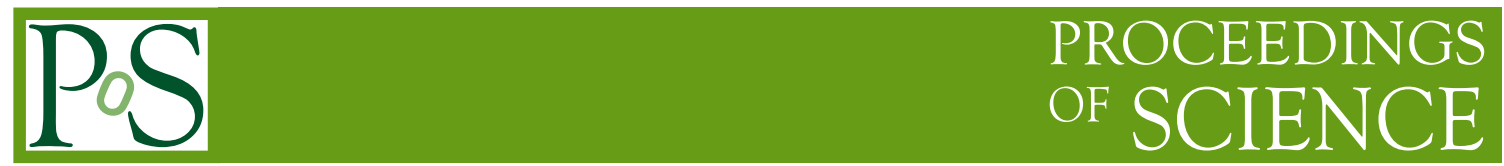

\title{
Geometry of the New Massive Gravity Domain Walls
}

\author{
U. Camara dS*, G. Sotkov ${ }^{\dagger}$ \\ Departamento de Física - CCE \\ Universidade Federal de Espirito Santo \\ 29075-900, Vitoria - ES, Brazil \\ E-mail: ulyssescamara@gmail.com
}

\begin{abstract}
The superpotential method for construction of flat static domain walls (DW's) solutions of threedimensional new massive gravity coupled to scalar matter is reviewed. The geometric properties of the asymptotic $A d S_{3}$ space-times representing DW's that relate two extrema of a particular (two degenerate minima) matter potential are described in details. The causal structure of such positive tensions DW's is discussed.
\end{abstract}

4th International Conference on Fundamental Interactions -ICFI2010,

August 1-7, 2010

Viçosa, Brazil

\footnotetext{
* Speaker.

†gsotkov@yahoo.com.br
} 


\section{Massive higher derivatives gravity}

The "higher derivatives" extensions of the Einstein gravity action involving squares of the curvature invariants: $R^{2}, R_{\mu v} R^{\mu v}$ and $R_{\mu v \rho \tau} R^{\mu v \rho \tau}$ naturally appear in the supergravity models derived from the (low energy) superstrings and also as 1-loop counter-terms in the perturbative quantization of Einstein gravity [1]. The corresponding equations of motions contain up to fourth order derivatives of the metrics $g_{\mu \nu}$ and a new mass scale $m^{2}$, say $R+\frac{1}{m^{2}} R^{2}$, related to the "graviton" mass. Including of such terms is known to lead to certain causality and unitarity problems [1] and to significant corrections to the Newton's gravitational potential as well [2]. The question arises whether one can find a particular combination of such terms that is free of the problems mentioned above. A natural candidate appears to be the "topological" Gauss-Bonnet term: $\mathscr{L}_{\mathscr{G} \mathscr{B}}=$ $R^{2}-4 R_{\mu v} R^{\mu v}+R_{\mu v \rho \tau} R^{\mu v \rho \tau}$, which is a total derivative in four dimensions. As is well known for conformally flat solutions, i.e. of vanishing $d>3$ Weyl tensor, the action of the Gauss-BonnetEinstein gravity becomes identical to the new massive gravity (NMG) one [3]:

$$
S=\frac{1}{\kappa^{2}} \int d^{d} x \sqrt{-g}\left\{\varepsilon R+\frac{1}{m^{2}}\left(R^{\mu v} R_{\mu v}-\frac{d}{4(d-1)} R^{2}\right)-\kappa^{2}\left(\frac{1}{2}|\vec{\nabla} \sigma|^{2}+V(\sigma)\right)\right\},
$$

where $\kappa^{2}=16 \pi G$ and $\varepsilon= \pm 1$. An important feature of this model is that for all $d>2$ the equations of motion for static domain wall (DW) solutions are of second order [6], independently of the form of the matter potential. Although the GB-term in three dimensions is identically zero, the action of $d=3 \mathrm{NMG}$ model (obtained from the Pauli-Fierz Lagrangian [3]) again has the form (1.1). As it was shown in ref.[3], the pure $d=3 \mathrm{NMG}$ gravity has the remarkable property to be ghost free (i.e. unitary) under certain restrictions on the values of the parameters. Its vacuum ( $\sigma=$ const) sector contains two massive degrees of freedom (the "graviton" polarizations) and unlike 3D Einstein gravity it admits physically interesting classical solutions - gravitational waves, black holes, solitons, etc. [4],[6],[5].

The present paper is reviewing the specific new features of the superpotental method when applied to $d=3$ NMG-mater model (1.1) (see [6]). We consider as an example a particular superpotential given by $W(\sigma)=B \cosh D \sigma$, which turns out to generate bounded Higgs-like matter potential $V(\sigma)$ with few extrema $\sigma_{A}^{*}$ : i.e. $V^{\prime}\left(\sigma_{A}^{*}\right)=0$ [8]. The use of the superpotential and related (BPS-like) first order equations are known to provide a vast family of exact solutions of the corresponding (2+1)-dimensional NMG model [5], [6], [7]. The most simplest of them are the vacuum solutions determined by the (stable) extrema $\sigma_{a, b}^{*}=$ const of $V(\sigma)$ and representing different $A d S_{3}$ space-times of (negative) cosmological constants $\Lambda_{a, b}=\frac{\kappa^{2}}{2} V\left(\sigma_{a, b}^{*}\right)$. We are further interested in the description of the geometric properties of a class of static flat DW's defined (as usually [11]) by the following ansatz:

$$
d s^{2}=d z^{2}+e^{\varphi(z)}\left(d x^{2}-d t^{2}\right), \quad \sigma=\sigma(z),
$$

together with the specific boundary conditions (b.c.): $e^{\varphi}(z \rightarrow \pm \infty) \approx e^{2 \sqrt{\left|\Lambda_{a, b}^{e f f}\right|}}$ and $\sigma( \pm \infty)=\sigma_{a, b}^{*}$, appropriate to the case of NMG models of at least two AdS-type vacua. Then the resulting DW's represent smooth solutions of the NMG-matter model interpolating between two such vacua configurations placed at the causal limits, i.e. $z \rightarrow \pm \infty$ ends (boundaries and/or horizons) of the effective 
asymptotically $A d S_{3}$ (called $(a) A d S_{3}$ ) space-times. Their main feature is that the matter energy is concentrated around certain $(d-2)$-dimensional subspace (i.e. a line for $d=3$ ) that divides the (a) $A d S_{3}$ space-time in two parts containing (asymptotically) different $A d S_{3}$ vacua $\left(\Lambda_{a, b}, \sigma_{a, b}^{*}\right)$ [11], [8].

As usually the investigation of such DW's is motivated by their important role in the description of the renormalization group (RG) flows and corresponding phase transitions in twodimensional QFT "holographically dual" to 3D massive gravity [9], [7] in the frameworks of $A d S_{3} / C F T_{2}$ correspondence [14].

\section{Domain Walls from Superpotential}

Due to the particular choice of the relative coefficient of the "quadratic" terms in the action, the equations of motions for flat static DW's (1.2) derived from $d=3$ NMG action (1.1) are of second order:

$$
\begin{aligned}
\ddot{\sigma}+\dot{\sigma} \dot{\varphi}-V^{\prime}(\sigma)=0, \quad \dot{\varphi}^{2}\left(1-\frac{\dot{\varphi}^{2}}{16 \varepsilon m^{2}}\right)+\varepsilon \kappa^{2}\left(-\dot{\sigma}^{2}+2 V(\sigma)\right) & =0, \\
\ddot{\varphi}\left(1-\frac{\dot{\varphi}^{2}}{8 \varepsilon m^{2}}\right)+\frac{1}{2} \dot{\varphi}^{2}\left(1-\frac{\dot{\varphi}^{2}}{16 \varepsilon m^{2}}\right)+\varepsilon \kappa^{2}\left(\frac{1}{2} \dot{\sigma}^{2}+V(\sigma)\right) & =0 .
\end{aligned}
$$

As in the case of DW's of the pure Einstein Gravity (i.e. the $m^{2} \rightarrow \infty$ limit of (1.1)) an effective method for construction of analytic non-perturbative solutions of eqs. (2.1) consists in the introduction of an auxiliary function $W(\sigma)$ called superpotential [5], [6], [9], [11] such that ${ }^{1}$ :

$$
\begin{aligned}
& \kappa^{2} V(\sigma)=2\left(W^{\prime}\right)^{2}\left(1-\frac{\kappa^{2} W^{2}}{2 \varepsilon m^{2}}\right)^{2}-2 \varepsilon \kappa^{2} W^{2}\left(1-\frac{\kappa^{2} W^{2}}{4 \varepsilon m^{2}}\right), \\
& \dot{\varphi}=-2 \varepsilon \kappa W, \quad \dot{\sigma}=\frac{2}{\kappa} W^{\prime}\left(1-\frac{\kappa^{2} W^{2}}{2 \varepsilon m^{2}}\right),
\end{aligned}
$$

where $W^{\prime}(\sigma)=\frac{d W}{d \sigma}, \dot{\sigma}=\frac{d \sigma}{d z}$ etc. Then for each given $W(\sigma)$ we find that all the solutions of the first order system (2.2) are solutions of the eqs. (2.1) as well.

The vacuum solutions of NMG gravity represent constant $\sigma$ solutions of eqs. (2.2) that are determined by the real roots of the following equations: (a) $W^{\prime}\left(\sigma_{a}^{*}\right)=0$ and (b) $W^{2}\left(\sigma_{b}^{*}\right)=\frac{2 \varepsilon m^{2}}{\kappa^{2}}$. Each one of them $A=a, b$ describes $A d S_{3}$ space, i.e. $d s^{2}=d z^{2}+e^{-2 \varepsilon \sqrt{\left|\Lambda_{e f f}^{A}\right|}}\left(d x^{2}-d t^{2}\right)$, of cosmological constant $\Lambda_{e f f}^{A}=-\kappa^{2} W^{2}\left(\sigma_{A}^{*}\right)$. These values of $\Lambda_{e f f}^{A}$ are obtained from 3D scalar curvature as follows:

$$
R=-2 \ddot{\varphi}-\frac{3}{2} \dot{\varphi}^{2} \equiv 8 \varepsilon\left(W^{\prime}\right)^{2}\left(1-\frac{\kappa^{2} W^{2}}{2 \varepsilon m^{2}}\right)-6 \kappa^{2} W^{2}
$$

and therefore we have $R_{v a c}=-6 \kappa^{2} W^{2}\left(\sigma_{A}^{*}\right)=6 \Lambda_{e f f}^{A}$. Observe that all the type (b) vacua have by construction equal cosmological constants $\Lambda_{e f f}^{(b)}=-2 \varepsilon m^{2}=-\frac{1}{L_{b}^{2}}$. It becomes clear that the variety of admissible vacua of NMG-matter model (1.1) is defined by the extrema of the potential $V(\sigma)$

\footnotetext{
${ }^{1}$ which represent an adapted $d=3$ version of $d=5$ Low-Zee superpotential [10] for the Gauss-Bonnet(GB) extended 5D gravity.
} 
and by the values of the parameters $\varepsilon$ and $m^{2}$. We are looking for DW's interpolating between two different $A d S_{3}$ vacua $\left(\sigma_{A}^{*}, \Lambda_{e f f}^{A}\right)$, parametrized by the solutions of the following algebraic equations: $V^{\prime}\left(\sigma_{A}^{*}\right)=0$ and $2 \Lambda_{e f f}^{A}\left(1+\frac{\Lambda_{e f f}^{A}}{4 \varepsilon m^{2}}\right)=\varepsilon \kappa^{2} V\left(\sigma_{A}^{*}\right)$.

Independently of the form of the superpotential $W(\sigma)$, another important property of all the DW's solutions is the following simple "total derivative" form of the NMG domain walls action:

$$
S_{N M G}(D W)=\frac{2 V_{0}}{\kappa} \int_{-\infty}^{\infty} \frac{d}{d z}\left[e^{\varphi} W(\sigma)\left(1+\frac{\kappa^{2} W(\sigma)^{2}}{2 \varepsilon m^{2}}\right)\right] d z, \quad V_{0}=\int d x d t
$$

As in the case of 3D Einstein (massless) gravity DW's [8],[9], [6] this form of $S_{N M G}(D W)$ is derived by substituting the $I^{s t}$ order equations (2.2) in the action of $d=3$ NMG-matter model (1.1). Note that the value of the action $S_{N M G}(D W)$ is infinite due to $2 D$ volume $V_{0}=\infty$ and to the divergent scale factor as well. These divergences also reflect the fact that for planar DW's the scalar matter is uniformly distributed along the whole $x$-axis and therefore such DW's have infinite energy. An important characteristics of the gravitational properties of such DW's is then given by the values of their energy densities $\varepsilon_{D W}=\frac{E_{D W}}{L_{x}}$ (equals of their tensions $\tau_{D W}$ ). In the case of $(a) A d S_{3}$ geometries it is given by [12]:

$$
\tau_{D W}=\lim _{L_{x} \rightarrow \infty} \frac{1}{L_{x}} \sum_{A= \pm} v_{A} \int_{-L_{x} / 2}^{L_{x} / 2} d x \xi^{i} T_{i j}^{(A)} \xi^{j}, \quad T_{i j}^{(A)}=-\frac{2}{\sqrt{-\gamma^{A}}} \frac{\delta S_{N M G}^{B Y}}{\delta \gamma_{A}^{i j}} v_{A}, \quad i, j=0,1
$$

where $A= \pm$ denote the two $z \rightarrow \pm \infty$ limits $(\partial M)_{A}$ describing $(a) A d S_{3}$ boundaries or/and horizons of metrics $\gamma_{i j}^{A}(x, t)=\lim _{z \rightarrow \pm \infty} \gamma_{i j}(x, t \mid z) ; v_{ \pm}= \pm 1$ and $\xi^{\mu}=\left(0, \xi^{i}\right)$ is time-like Killing vector, orthogonal to both $(\partial M)_{A}$ - surfaces and normalized as $\xi^{i} \gamma_{i j}^{A} \xi^{i}=-1$. The $T_{i j}^{(A)}$ is the Brown-York (B-Y) "boundary" stress-tensor [12]. Since for pseudo-Riemannian manifolds with boundaries and/or horizons (due to non-trivial b.c.'s) the action (1.1) has not well-posed variational problem, an appropriate surface term (similar to the Gibbons-Hawking one $\frac{2}{\kappa} \int d^{2} x \sqrt{-\gamma} K$ ) must be added to $S_{N M G}$. For the case of pure NMG gravity (i.e. action (1.1) with $\sigma=$ const) such terms have been introduced by Hohm and Tonni [13]:

$$
S_{g G H}=-\frac{2}{\kappa^{2}} \sum_{A= \pm} v_{A} \int_{(\partial M)_{A}} d x d t \sqrt{-\gamma}\left(\varepsilon K-\frac{1}{2} f K+\frac{1}{2} f_{i j} K^{i j}\right)
$$

where $K_{i j}$ is the extrinsic curvature of $2 \mathrm{D}$ surface $(\partial M)_{A} ; f_{\mu \nu}$ is the auxiliary Pauli-Fierz spin two field [3], [13] whose "on-shell" form used in eq. (2.6) is given by $f_{\mu \nu}=\frac{2}{m^{2}}\left(R_{\mu \nu}-\frac{1}{4} g_{\mu \nu} R\right)$ with $\mu, v=0,1,2$ and $f=\gamma^{i j} f_{i j}, K=\gamma^{i j} K_{i j}$. In the case of DW's (1.2) we can use eqs. (2.2) in order to get the following "boundary" form of the improved $S_{N M G}^{B Y}=S_{N M G}+S_{g G H}$ action :

$$
S_{N M G}^{B Y}(D W)=-\frac{2}{\kappa} \sum_{A= \pm} v_{A} \int_{(\partial M)_{A}} d x d t \sqrt{-\gamma} W(\sigma)\left(1+\frac{\kappa^{2} W^{2}(\sigma)}{2 \varepsilon m^{2}}\right)
$$

Then according to the definitions (2.5) and (2.6) one can easily derive the boundary (B-Y) stresstensor for the $d=3$ NMG-matter model(1.1) model and the corresponding finite values of the DW's tensions as well:

$$
T_{i j}^{A}(D W)=-\frac{2}{\kappa} W\left(\sigma_{A}^{*}\right)\left(1+\frac{\kappa^{2} W^{2}\left(\sigma_{A}^{*}\right)}{2 \varepsilon m^{2}}\right) \gamma_{i j}^{A}, \quad \tau_{D W}=\frac{2}{\kappa} \sum_{A= \pm} v_{A} W_{A}\left(1+\frac{\kappa^{2} W_{A}^{2}}{2 \varepsilon m^{2}}\right)
$$


where $W_{A}=W\left(\sigma_{A}^{*}\right)$. Note that in the $m^{2} \rightarrow \infty$ limit the above formula reproduces the well know results for a flat DW's tensions in 3D Einstein gravity obtained by the Israel's thin wall approximation [11].

\section{On the geometry of DW's}

We are looking for DW's of $d=3$ NMG model (1.1) of Higgs-like (two equal minima) matter potential interpolating between two different $A d S_{3}$ vacua $\left(\sigma_{A}^{*}, \Lambda_{\text {eff }}^{A}\right)$. The simplest superpotential that carries all the desired properties is given by $W=B \cosh D \sigma$. For this choice of $W(\sigma)$ we always have one type $(a)$ vacuum at $\sigma=0$ and of negative cosmological constant $\Lambda_{e f f}^{a}=-\kappa^{2} B^{2}$ (i.e. $L_{a}=\frac{1}{\kappa B}$ ). We next consider the case when: $B>0, D>0$ and $\varepsilon=-1, m^{2}<0$. Depending on the values of the parameter $B$ (i.e. of $L_{a}$ ) we can have few type (b) vacua:

$$
\sigma_{b}^{ \pm}=\frac{1}{D} \ln \left(\frac{L_{a}}{L_{b}} \pm \sqrt{\frac{L_{a}^{2}}{L_{b}^{2}}-1}\right), \quad \sigma_{b}^{+}=-\sigma_{b}^{-},
$$

(all of equal cosmological constants $\Lambda_{e f f}^{b}=-\frac{1}{L_{b}^{2}}$ ), namely: (1) two $b$-vacua when $L_{b}<L_{a}$; (2) one (degenerate) $b$-vacuum for $L_{b}=L_{a}$ and (3) no one $b$-vacuum when $L_{b}>L_{a}$. Due to the reflection symmetry $\sigma \rightarrow-\sigma$ of the superpotential we further restrict ourselves to consider DW's in the regions corresponding to $\sigma>0$ only. Then the region $(1+)$ contains two $A d S_{3}$ vacua $\sigma_{a}=0$ and $\sigma_{b}^{+}$dividing it in two parts: $0<\sigma<\sigma_{b}^{+}$and $\sigma_{b}^{+}<\sigma<\infty$. The corresponding DW's solutions are obtained by direct integration of the first order system of eqs. (2.2):

$$
e^{2 \varepsilon y_{a}\left(\frac{z}{L_{a}}\right)}=\left(\frac{\cosh D \sigma-1}{\cosh D \sigma+1}\right)\left(\frac{L_{b} \cosh D \sigma+L_{a}}{L_{b} \cosh D \sigma-L_{a}}\right)^{\frac{L_{b}}{L_{a}}}, \quad e^{\varphi-\varphi_{0}}=\left(\frac{L_{b}^{2} \cosh ^{2} D \sigma-L_{a}^{2}}{\cosh ^{2} D \sigma-1}\right)^{\frac{2}{y_{a}}}
$$

where we have introduced new parameters $y_{a}=-\frac{y_{b}}{2}=\frac{2 \varepsilon D^{2}}{\kappa^{2}}\left(1-\frac{L_{b}^{2}}{L_{a}^{2}}\right)$. They naturally appear in the asymptotic forms of $\sigma(z)$, say for $\varepsilon=-1$ :

$$
\sigma(z) \stackrel{z \rightarrow \infty}{\approx} \sigma_{b}^{+}-\sigma_{b}^{0} e^{-y_{b} \sqrt{\left|\Lambda_{e f f}^{b}\right| z}}, \quad \sigma(z) \stackrel{z \rightarrow-\infty}{\approx} \sigma_{a}-\sigma_{a}^{0} e^{-y_{a} \sqrt{\left|\Lambda_{e f f}^{a}\right|}}
$$

where $\sigma(\infty)=\sigma_{b}^{+}, \sigma(-\infty)=\sigma_{a}$ and $\sigma_{a}^{0}, \sigma_{b}^{0}$ are certain constants determined by eqs. (3.2). For example we have $\sigma_{a}^{0}=\frac{2}{D}\left(\frac{L_{a}-L_{b}}{L_{a}+L_{b}}\right)^{\frac{L_{b}}{2 L_{a}}}$. It is worthwhile to mention that the $y_{A}$ 's determine the scaling dimensions $\Delta_{A}=2-y_{A}$ of the $2 d$ field $\Phi_{A}(x, t)$ dual to the $3 d$ bulk field $\sigma(z \mid x, t)$. According to the $A d S_{3} / C F T_{2}$ correspondence principle for each NMG vacua $\left(\sigma_{A}, \Lambda_{e f f}^{A}\right)$ they are given in terms of the corresponding "effective" $\sigma$-field masses $m_{A}^{2}=V^{\prime \prime}\left(\sigma_{A}\right)$ as follows: $m_{A}^{2} L_{A}^{2}=y_{A}\left(y_{A}-2\right)$.

The asymptotic behaviour of the scale factor $e^{\varphi(z)}$ in the case of $\varepsilon=-1$ :

$$
e^{\varphi z \rightarrow \infty} \stackrel{2 \sqrt{\left|\Lambda_{e f f}^{b}\right|}}{=} \rightarrow \infty, \quad e^{\varphi \stackrel{z \rightarrow-\infty}{=}} e^{2 \sqrt{\left|\Lambda_{e f f}^{a}\right|}} \rightarrow 0
$$

indicates that the above DW's solution (3.2) is interpolating smoothly between two $A d S_{3}$ - type (a) and type (b) vacua - and it represents particular $(a) A d S_{3}$ space-time. The divergence of the scale factor at $z \rightarrow \infty$ corresponds to $A d S_{3}$ type of boundary of cosmological constant $\Lambda_{\text {eff }}^{b}$, while 
the region $z \rightarrow-\infty$ of vanishing scale factor describes null Cauchy horizon, where the causal description in the Poincare patch terminates. It becomes evident that the near horizon region can be (asymptotically) described as another $A d S_{3}$ of cosmological constant $\Lambda_{e f f}^{a}$, i.e. representing the type $(a)$ NMG vacua $\sigma_{a}=0$.

The properties of the solution for $\sigma \in\left[\sigma_{b}^{+}, \infty\right)$ are rather different. For $z \rightarrow \infty$ we recover the same "boundary" behaviour as the one corresponding to vacuum $(b)$ and given by eqs. (3.3) and (3.4). We find however that $\sigma$ is divergent at $z=0$ (i.e. $\sigma(0)=\infty$ ) with infinite scalar curvature, indicating that now causal description is terminating at $z=0$ with naked singularity.

In order to describe the causal structure of the $(a) A d S_{3}$ space-times representing regular DW's solutions (i.e. $0<\sigma<\sigma_{b}^{+}$) of the NMG-matter model relating two different $A d S_{3}$ vacua it is convenient to introduce new coordinates:

$$
v=\int^{z} d z^{\prime} e^{-\frac{\varphi\left(z^{\prime}\right)}{2}}+\text { const }, \quad d s^{2}=\Omega^{-2}(v)\left(d v^{2}+d x^{2}-d t^{2}\right), \quad \Omega(v)=e^{-\frac{\varphi(z(v))}{2}}, .
$$

We can further choose the arbitrary constant above such that $v$ to be defined on the half-line, i.e. $0<v<\infty$. Even without the knowledge of the explicit form of the conformal factor $\Omega^{2}(v)$ it is evident that this DW space-time has causal structure similar to the one of a "half" of the Minkowski space $M_{3}$, i.e. the one having the plane $v=0$ as its boundary. It is therefore necessary to introduce appropriate global coordinates (say for fixed $x$ ) $-\infty<\tau<\infty$ and $0<\theta<\pi$

$$
v=\frac{1}{2} \frac{\sin \theta}{\cos \left(\frac{\tau+\theta}{2}\right) \cos \left(\frac{\tau-\theta}{2}\right)}, \quad t=\frac{1}{2} \frac{\sin \tau}{\cos \left(\frac{\tau+\theta}{2}\right) \cos \left(\frac{\tau-\theta}{2}\right)}, \quad d s^{2}=\frac{e^{\varphi(\theta, \tau)}\left(d \theta^{2}-d \tau^{2}\right)}{4 \cos \left(\frac{\tau+\theta}{2}\right) \cos \left(\frac{\tau-\theta}{2}\right)}
$$

Then the asymptotic behaviour of the metrics near the ends of the interval $v \in(0, \infty)$, namely

$$
\begin{aligned}
& d s^{2} \approx \frac{1}{\Lambda^{(b)}} \frac{1}{\sin ^{2} \theta}\left(d \theta^{2}-d \tau^{2}\right), v \rightarrow \infty, \theta \pm \tau \rightarrow(2 n+1) \pi,-(2 n+1) \pi, n=0,1, \ldots \\
& d s^{2} \approx \frac{1}{\Lambda^{(a)}} \frac{1}{\sin ^{2} \theta}\left(d \theta^{2}-d \tau^{2}\right), v \rightarrow 0, \theta \rightarrow 0, \pi
\end{aligned}
$$

demonstrates that we indeed have two different $A d S_{3}$ spaces representing the regions in the neighbourhoods of the boundary and of the Cauchy horizon, as one can see from the corresponding Penrose diagram shown at Fig.1.

\section{Conclusions}

The superpotential method described in Sect.2. (see also [6] for more details) provides an effective tool for explicit construction of DW's solutions of the NMG-matter model (1.1) for any given superpotential $W(\sigma)$ that admits at least two $A d S_{3} \mathrm{NMG}$ vacua $\left(\sigma_{A}, \Lambda_{\text {eff }}^{A}\right)$. As we have shown in Sect. 3 on the particular example of $W=B \cosh D \sigma$, the proper existence of such vacua (and of DW's between them) imposes certain restrictions on the shape of the corresponding matter potential $V(\sigma)$, namely: $\varepsilon m^{2}>0$ and $L_{b}<L_{a}$ or equivalently $2 \varepsilon m^{2}>\kappa^{2} B^{2}$. Under these conditions and when $\varepsilon=-1$ and $m^{2}<0$, we observe that the parameters $y_{A}$ defining the $\sigma$ asymptotics are of opposite signs: $y_{a}<0$ and $y_{b}>0$. As a consequence the $2 d$ operators $\Phi_{A}$ duals to $\sigma$ have rather different properties: $\Phi_{b}$ is relevant operator, while the $\Phi_{a}$ is irrelevant one, as required in order that such DW represents an RG flow in $2 d$ perturbed CFT dual to NMG-matter model. Since 


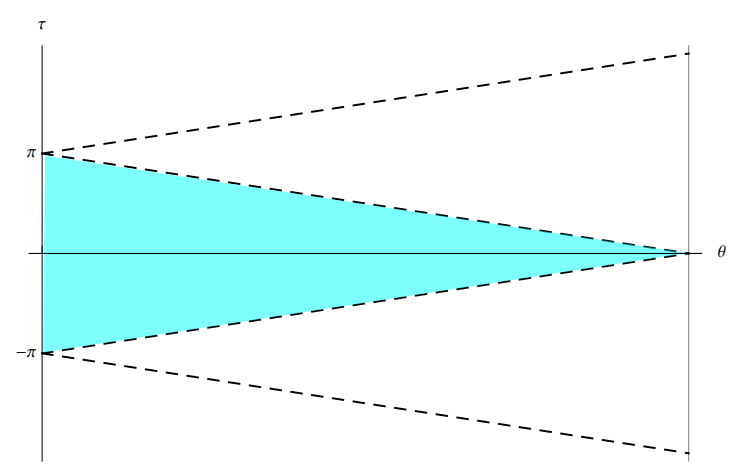

Figure 1: Reduced Carter-Penrose diagram of DW's space-time: the shaded region represents the original Poincare patch with $\theta=0, \pi$ lines corresponding to $\operatorname{Ad}_{3}\left(\Lambda_{\text {eff }}^{b}\right)$ boundary, while the dotted lines to the Chauchy horizon of near-horizon $A d S_{3}\left(\Lambda_{\text {eff }}^{a}\right)$ metrics.

in this case we also have $m_{a}^{2}>0$, one can conclude that the vacuum $(a)$ is always a minima of $V(\sigma)$. It turns out that the type $(b)$ vacuum represents one maxima of $V(\sigma)\left(\right.$ i.e. $\left.m_{b}^{2}<0\right)$ only if further conditions are added, namely: $0<y_{b}<2$ which leads to the following restrictions on the parameters of the model: $1-\frac{\kappa^{2}}{2 D^{2}}<\frac{L_{b}^{2}}{L_{a}^{2}}<1$. Let us mention that in this case the BreitenlohnerFreedman unitarity condition [15] for scalar field in $A d S_{3}$ background: $\Lambda_{e f f}^{b}<m_{b}^{2}<0$ is indeed also satisfied. The complete description of DW's properties includes also the values of their energy densities and tensions $\tau_{D W}=E_{D W} / L_{x}$. According to eq. (2.8) we find that the tensions:

$$
\tau_{D W}\left(L_{b}, L_{a}\right)=\frac{2\left(L_{a}-L_{b}\right)}{\kappa^{2} L_{a} L_{b}}\left(2+\left(L_{a}+L_{b}\right) \frac{L_{b}}{L_{a}^{2}}\right)>0 .
$$

of the regular DW's we have constructed in Sect. 3 above are always positive.

It is worthwhile to mention in conclusion that the methods for construction of DW solutions of the NMG-matter model reported in the present paper provide a set of important ingredients essentials in the description of the RG flows and phase transitions that occur in the corresponding dual 2d non-conformal QFT's [8], [9], [7].

Acknowledgments We are grateful to C.P. Constantinidis for the discussions and for critical reading of the manuscript. This work has been partially supported by PRONEX project number 35885149/2006 from FAPES-CNPq (Brazil).

\section{References}

[1] G.t'Hooft and M.Veltman, Ann.Inst.H.PoincareA20(1974),69-94; K.S.Stelle,Phys.Rev.D16(1977)953-968.

[2] I.Gullu adn B.Tekin, Phys.Rev.D 80:064 033(2009).

[3] E.A. Bergshoeff, O. Hohm and P.K. Townsend.,Phys. Rev. Lett.102, 201301(2009);

Phy. Rev. D79 124042/2009; Gravitons in Flatland, arXiv:1007.4561.

[4] G.Clement,Class.Quant.Grav.26,105015(2009),Phys.Rev.Lett.102,201301(2009); E.Ayon-Beato,G.Giribet,M.Hassaine,JHEP0905,029 (2009);J.Oliva,D.Tempo and R.Troncoso,JHEP 0907:011(20009);H.Ahmedov and A.N.Aliev,arxiv:1008.0303. 
[5] H.L.C. Louzada, U. Camara dS and G.M. Sotkov, Phys. Lett. 686 B (2010) 268.

[6] U. Camara dS and G.M. Sotkov, Phys. Lett. 694 B (2010)94-99.

[7] U.Camara dS,C.P.Constantinidis,G.M.Sotkov,New Massive Gravity Holography, arXiv:1009.2665.

[8] L.Girardello,M.Petrini,M.Porrati and A.Zaffaroni,Nucl.Phys.B569 (2000)451.

[9] D.Z.Friedman,C.Nunez,M.Schnabl,K.Skenderis,Phys.Rev.D69,104 027(2004).

[10] I.Low and A.Zee, NPB585,(2000),395-401.

[11] M. Cvetic and H.H. Soleng, Phys. Rep. 282(1997) 159 and references therein.

[12] J.D.Brown and J.W. York, Phys. Rev.D47, 1407 (1993).

[13] O. Hohm abd A. Tonni, arXiv: 1001.3598.

[14] J.Maldacena,Adv.Theor.Math.Phys.2:231(1998);S.S.Gubser,I.R.Klebanov, A.Polyakov,Phys.Lett.428 B(1998)105E.Witten,Adv.Theor.Math.Phys.2:253(1998).

[15] P.Breitenlohner and D.Z.Freedman,PLB115(1982),197;Ann.Phys.144(1982)249. 